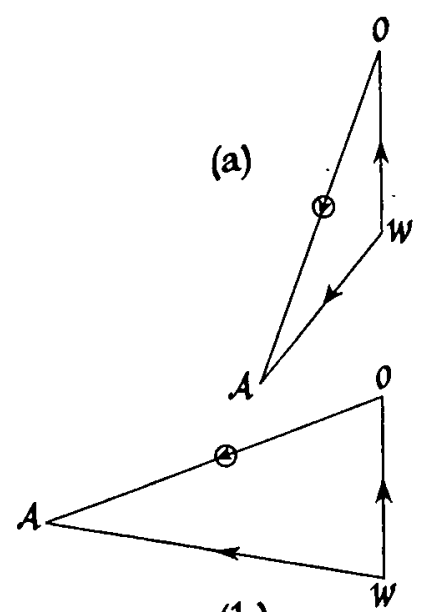

(b) (c)

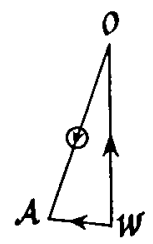

(d) (e)

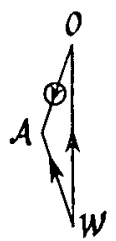

(f)

Fig. 3

occupants of the bridge in foggy weather, and between them they must carry out the manifold duties prescribed by law and the ordinary practice of seamen. It is inevitable that radar plotting and observing are not carried out with maximum efficiency. In such cases this plotting device shows to best advantage and can be used by the observer who, as a practical and experienced navigator, can interpret the information given and decide on the appropriate action to avoid collision.

In the ordinary course of general trading on the regular shipping routes of the world, the great majority of vessels sighted will be on or near parallel courses and most of them will pass at a safe distance. In such cases only the plotting of bearing and distance is required to show that the ship will pass clear. When the occasional ship is found to be approaching on or near a collision course it can be given full attention, and a decision quickly made as to the action required to keep out of the region of collision, probably by a bold alteration of course.

\title{
Navigation Using the Transit of an Artificial Satellite
}

\author{
from D. H. Shinn \\ (Research Division, Marconi's W/T Co.)
}

F. G. SMITH describes (this Journal, 13, 109) a system for determining position from a single observation, probably lasting for a time of the order of a few minutes, of radio transmissions from an artificial Earth satellite. There are in this system two engineering difficulties which I think Dr. Smith has underestimated; these are the frequency stability and the power required. In order to establish these requirements it is necessary to consider in some detail how Dr. Smith's system would work. 
He suggests that a satellite might be launched on a nearly circular orbit at a height of 400 nautical miles and an inclination to the equator of $64^{\circ}$, that it would transmit a continuous signal on, say, $300 \mathrm{Mc} / \mathrm{s}$. for some years, and that predictions of its position, accurate to about a mile, be issued at suitable intervals.

The observer would measure the frequency of the received signal. He would have to find the time at which the frequency received was the same as the frequency transmitted, and the rate of change of frequency at this time. This could be done conveniently by noting the times at which the frequency was equal to certain previously chosen values, and computing therefrom the two parameters required; as much of this process as convenient could be made automatic.

As Dr. Smith explains, if the satellite flies overhead or nearly overhead, it is useless for navigational purposes. If this happens, the observer must wait for the next transit 100 minutes later. Now if the observer is on or near the equator the minimum distance of the satellite from him during this transit is 1600 nautical miles. It follows that the rate of change of frequency at this point is about 16 cycles per second per second (assuming a transmitted frequency of $300 \mathrm{Mc} / \mathrm{s}$.). Since it is necessary to measure the time at which the frequency received is the same as the frequency transmitted to an accuracy of 0.2 seconds, it is necessary to know the absolute value of this frequency, and for the observer himself to have an absolute standard of frequency, the maximum allowable error in the comparison being $3 \mathrm{c} / \mathrm{s}$., i.e. each frequency must be known to better than $\mathrm{I}$ in 10.8

Frequency standards of this accuracy, based on quartz crystal oscillators, could be produced in quantity and supplied to ships, at a price. Regular checking would have to be carried out, and this could only readily be done when the ship was in port and had access to very accurate time or frequency standards ( 1 in $10^{8}$ is equal to about $0.00 \mathrm{I}$ seconds per day). Ensuring the stability of the oscillator during an ocean voyage, when no check will be possible, would be a challenging engineering problem. Of course, if the frequency did change, unknown to the operator, serious navigational errors might result.

In order to check the frequency on board ship, it has been suggested ${ }^{3}$ that the satellite should itself transmit time signals at times known to a fraction of a millisecond. These time signals would be compared with those generated by the shipborne oscillator, and the difference found to a fraction of a millisecond. The shipborne oscillator could then be calibrated daily. For satisfactory operation both the satellite-borne and the shipborne oscillators should be stable to a few parts in $10^{9}$ per day. Sufficient accurate information about the satellite's orbit and frequency could also be transmitted from the satellite at suitable intervals; this information could be corrected daily. The shipborne equipment would be complicated to make, to service, and to use, but it is within the range of present techniques. However, if during an ocean voyage the power supply failed, or an electronic fault occurred, it might be difficult to reset the frequency to the required accuracy during the voyage. This difficulty could be partially met by carrying two oscillators.

The same problem of stability exists within the satellite, and the problem of reliability is more serious here. It is worth recalling that the frequency of Sputnik I probably changed by about $I$ in $10^{6}$ during each orbit ${ }^{1,2}$; this is what would be expected from a crystal without very careful temperature control.

It may prove possible with such a system to use crystal-controlled oscillators in both satellite and ship. However, it would be worth while to investigate the possibility of using the more stable atomic or molecular frequency standards. 
These would have sufficient inherent stability to function without daily checking, but it would take several years to develop sufficiently reliable versions, and the ultimate cost cannot be predicted. The American space programme apparently includes development of such standards. 5 The Americans have so far launched two navigational satellites, but I have seen no account of what frequency standards they are carrying.

It has been reported 2,3 that it is possible to find the time of closest approach accurately from a study of the shape of the frequency-time curve, without knowledge of the absolute frequency. This, however, would require complicated recording and computing equipment, and would be basically impossible for distant transits, e.g. for a satellite whose closest approach is 1600 nautical miles, since only a small portion of this transit is above the radio horizon, and its maximum altitude is only $2^{\circ}$.

This last conclusion seems to conflict with a statement of Guier and Weiffenbach $^{3}$; they do not state the maximum distance at which analysis of the shape of the frequency-time curve gives the required accuracy, and this matter clearly deserves further investigation. The advisability of using a higher satellite, e.g. at a height of 1000 miles, also deserves attention, particularly if it is not intended to use one's knowledge of the absolute frequency radiated.

Dr. Smith does not mention the signal to noise ratio required. If it is required to achieve a signal to noise ratio of $20 \mathrm{db}$. for a range of 1600 nautical miles with an aerial gain of $-2 \mathrm{db}$. at each end, then the transmitter power required is 60 watts (assuming a bandwidth of $20 \mathrm{kc} / \mathrm{s}$., and a receiver noise factor of $4 \mathrm{db}$.). The continuous radiation of such a power from a satellite would require quite an advance in satellite technology.

A method of reducing the power required is to use special aerials for reception, e.g. a broadside array of eight half-wave dipoles, giving a gain of i $\mathrm{d} d \mathrm{~b}$. at its maximum; this aerial would have to be completely adjustable in azimuth and elevation. The power required in the satellite would then be reasonable, but the receiving aerial installation would require some space, and the system would be completely unusable by aircraft.

An alternative is to use a complicated receiving system such as Microlock. 4 This could use an effective bandwidth of only a few cycles per second; the transmitted power required would then be only a few tens of milliwatts.

In short, while Dr. Smith's system might well lead eventually to a very useful navigational aid, it would be wrong to suggest that, if only someone gave us a satellite, we could immediately set about using it for this purpose. Considerable engineering development would be required, and there seems little doubt that the shipborne (or airborne) equipment would be much more complicated than an ordinary communications receiver.

I should like to thank the Engineer-in-Chief of Marconi's Wireless Telegraph Co. Ltd. for permission to publish this note.

\section{REFERENCES}

1 Lea, N. (1957). Further radio observations of the first satellite, Nature, Lond., 180, 943.

2 Stanesby, H. and others (1958). Precise frequency measurements on the first Russian satellite; Analysis of doppler data from Earth satellites and discussion, Proc. Inst. Elect. Engineers, $105 \mathrm{~B}, 96-100$ and $108-112$. 
3 Guier, W. H. and Weiffenbach, G. C. (1960). A satellite Doppler Navigation System, Proc. Inst. Rad. Engrs., 48, 507.

4 Ashbrook, F. M. and Stevenson, D. D. (1960). The Navy's Portable Satellite Tracking Stations, Trans. Inst. Rad. Engrs., SET-6, 41 .

\title{
Dr. F. Graham Smith comments:
}

It is gratifying to note that the proposal for navigation by observation of an artificial satellite is being examined in more detail than in my paper. I certainly do not wish to take issue with Dr. Shinn about the rather more stringent requirements outlined in his note, except to comment that any relaxation of them leads only to a proportionate decrease of final accuracy rather than to a breakdown of the system.

The practical difficulties are in my opinion overestimated. Firstly, the transit satellites are already achieving a stability better than I part in I08. Secondly the shipborne equipment could match this at a cost of a few hundred pounds only. Thirdly the Microlock receiver is not a complicated device, and the stringent requirements on transmitter power and receiver sensitivity need not bother us.

It is hard to see reason for further hesitation when the transit system is such an obvious success, and when it is clear that a network of transit satellites will actually be in orbit in a few years' time.

\section{Dr. Shinn since writing his note has added (in proof) the following} comment :

Some of my cautionary remarks are outdated by the remarkable success of the transit satellites. It appears that a practically useful system will soon be operational, and that the problems of making the shipborne equipment sufficiently cheap and reliable for general use deserve detailed investigation.

\section{The Air Traffic Control Problem}

\author{
from A. W. Southall \\ (Wing Commander, R.A.F., retd.)
}

ON page 240 of the April Journal, Wing Commander Dickie pointed out that in traffic control as we know it today, it is ground-speed and not air-speed that really matters. As he says, it is not possible for the pilot to maintain a constant groundspeed and it is for this reason that I suggest the concept whereby individual aircraft maintain constant power which, in the short term, must give constant air-speed. Slight changes in power will maintain a planned traffic pattern on any one route at any one height. Individual speeds will of course be different, and overtaking will have to be arranged by divergencies of track or height.

Where traffic streams converge in either the vertical or the horizontal plane, an interlace is obviously necessary. However, provided clearances are restricted 